\title{
Promoting Affordable and Quality Tuberculosis Testing in India
}

uberculosis (TB) remains one of India's biggest health problems. Every year, India reports over 2 million TB cases. With the emergence of severe forms of drug-resistant TB and concerns about TB drug shortages, there is much work to be done to control the epidemic. ${ }^{[1]}$

The Revised National Tuberculosis Control Programme (RNTCP) has made good progress by providing basic TB diagnosis and treatment free of cost to all patients in the public sector. Recently, the RNTCP announced "universal access to quality TB diagnosis and treatment for all TB patients in the community" as its new goal for the next 5-year plan. ${ }^{[2]}$ This is a worthy goal, but any plan to reach all TB patients in India will need to include India's dominant private sector.

Why is the private health sector critical? In general, more than $80 \%$ of India's health care is delivered through the private sector. Most poor people who develop a cough first seek care in the informal private sector (chemists and unqualified practitioners), then from qualified practitioners. ${ }^{[3]}$ Ultimately, about $50 \%$ of them end up in the public sector where they receive free treatment. ${ }^{[4]}$ This pathway to curative care can take from weeks to months, during which patients continue to transmit infection to others. This delay, coupled with the high cost of care in private sector, drives many poor families into debt. And yet, for all the money spent, patients frequently undergo inaccurate TB tests and inappropriate TB drug treatment. ${ }^{[5,6]}$ Consequently, promptly getting patients the right test in the private sector is a critical first step for interrupting transmission and reducing the risk of drug resistance.

Unfortunately, TB testing practices in the private sector are completely different from those in the public sector. A majority (more than $90 \%$ ) of TB

\begin{tabular}{|l|l|}
\hline \multicolumn{2}{|c|}{ Access this article online } \\
\hline Quick Response Code: & Website: \\
\hline & www.jponline.org \\
\hline & \\
\hline
\end{tabular}

tests done by RNTCP are sputum smears. Diagnosis in the private sector is characterized by overuse of unreliable blood tests, low availability, and high cost of reliable quality-assured diagnostics tools, preference of blood as a sample, inability of the providers to separate the good from the bad tests, and the commercial incentives that inflate cost to the patients. ${ }^{[5,7]}$

Worldwide, sputum is the most important sample for diagnosis of lung TB and every guideline recommends the use of sputum-based tests. But, for several reasons, including poor regulation and financial incentives, blood is the most popular sample in the Indian private sector. ${ }^{[5]}$ Blood-based antibody tests are not accurate and discouraged by the World Health Organization (WHO). ${ }^{[8,9]}$

India's diagnostic landscape was abruptly disrupted in June of 2012 when the Government of India, acting on the 2011 WHO policy against serological tests, banned the use, import, sale, and manufacture of antibody-based blood tests for TB, and discouraged the use of tests like "TB Gold" for active TB [Figure 1]. This historic ban has had a big impact in reducing the use of inaccurate serological tests in India. However, the ban on serological tests has resulted in an unintended consequence - some laboratories have replaced antibody tests with unproven in-house polymerase chain reaction (PCR) or interferon-gamma release assays (IGRAs).

Blood PCR tests are unreliable and not recommended by any agency. There are acceptable blood tests (such as QuantiFERON-TB Gold, marketed in India as "TB Gold") for latent TB infection (which is treated with 6-9 months of isoniazid, to prevent progression from latent infection to active disease). These latent TB tests, however, are not recommended for active $\mathrm{TB}$ diagnosis by the WHO. ${ }^{[10]}$ Use of IGRAs for active TB will result in unacceptably high rates of false-positive results because IGRAs, like the Mantoux tuberculin skin test, cannot separate latent TB infection from active TB disease, and a large proportion of the Indian population is latently infected. ${ }^{[11]}$

Since the serology ban created a void in the market, it is important to address this gap and make sure that WHO-endorsed, sputum-based TB tests replace the inappropriate blood tests in the private sector. There are four accepted sputum tests that are recommended by the WHO and these are also used by the RNTCP. These are sputum smears, Xpert MTB/RIF, line probe assay, and liquid cultures. 


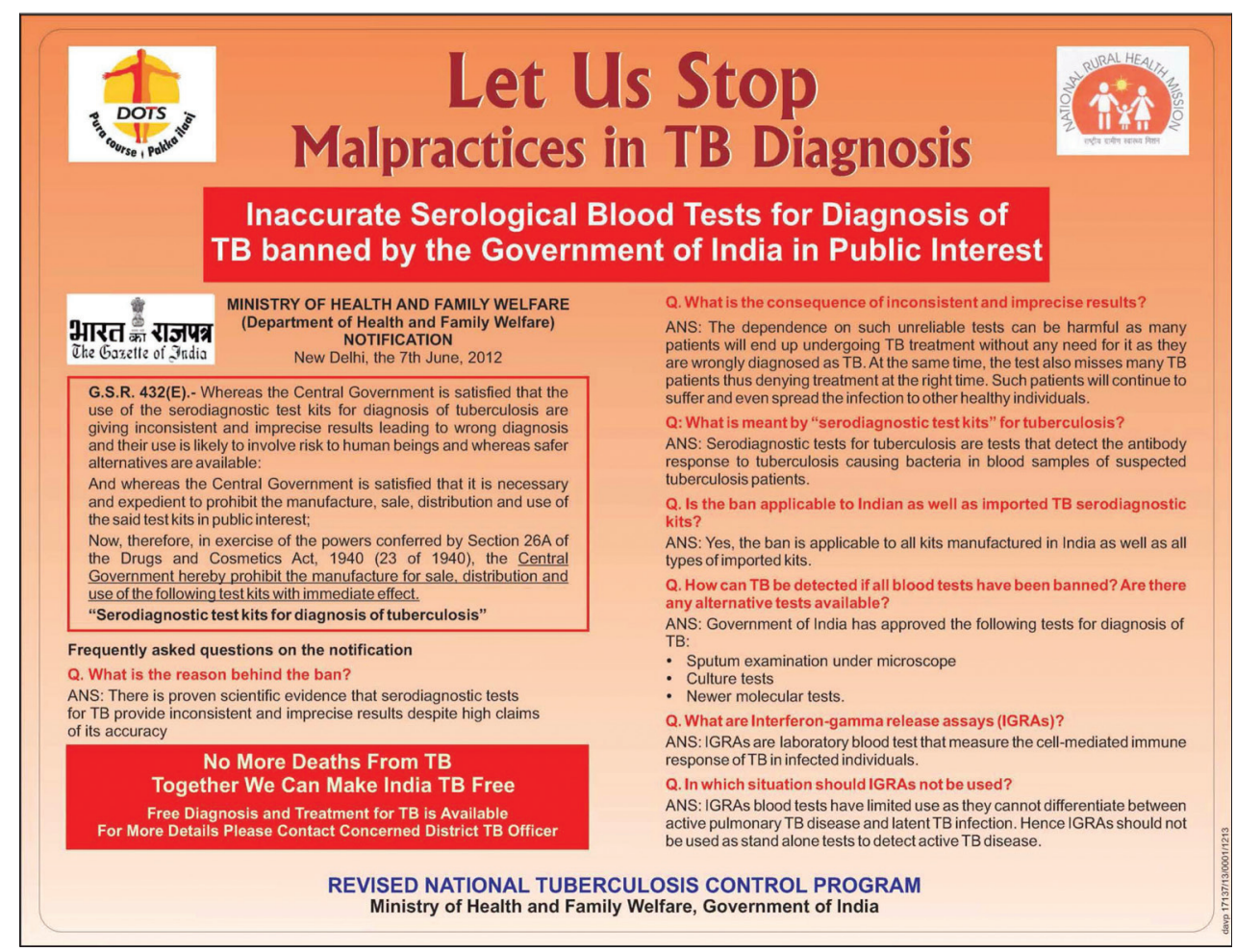

Figure 1: Advertisement on the ban on tuberculosis serological tests, published by the Revised National Tuberculosis Control Programme in leading Indian newspapers in 2012 (reproduced with permission from the Central Tuberculosis Division, RNTCP)

Although not highly sensitive, smear microscopy test is still very useful (and cheap) because it can rapidly identify the most infectious patients, and it is simple enough to be done in peripheral laboratories. Microscopy is under used in the private sector and this needs to change.

Recently, the WHO endorsed a new, rapid, automated, 2-hour molecular test called Xpert MTB/RIF, based on the GeneXpert platform (Cepheid Inc, USA) which can diagnose TB with great accuracy and can also detect those with drug resistance. ${ }^{[12]} \mathrm{A}$ recent Cochrane review has shown that the Xpert MTB/RIF test has $88 \%$ sensitivity and $98 \%$ specificity when compared to culture. ${ }^{[13]}$ Xpert MTB/RIF can detect rifampicin resistance with a sensitivity of $94 \%$ and specificity of $98 \% \cdot{ }^{[13]}$ Data from many countries, including India, clearly show substantially better performance of the Xpert MTB/RIF test over conventional smear microscopy. ${ }^{[13]}$ Emerging data also suggest that Xpert MTB/RIF has value for extrapulmonary TB (EPTB), especially TB lymphadenitis and TB meningitis. A WHO policy on use of Xpert MTB/RIF for EPTB and childhood TB is expected this year.

Another WHO-endorsed rapid molecular test, the line probe assay (e.g., Genotype MTBDRplus by Hain Lifescience, Germany) can also detect resistance to isoniazid (INH) and rifampicin with high accuracy and allow for rapid initiation of MDR-TB treatment, while waiting for liquid culture and drug susceptibility testing (DST). ${ }^{[14]}$ Last, liquid cultures (e.g., MGIT by BD, USA, and BacT/Alert by BioMerieux, France) are considered the gold standard for TB diagnosis and the only technology that can detect resistance to all major TB drugs. Liquid cultures are also very useful for smear-negative TB and extrapulmonary TB (EPTB).

If private physicians and laboratories replace suboptimal tests with the above sputum tests, this should greatly help improve the accuracy of TB diagnosis for patients in the country. The challenge is that good tests like GeneXpert, line probe assay and liquid culture are very expensive in the private sector. For example, the GeneXpert test can cost the patient as much as Rs. 3500 or higher in private laboratories. This is because WHO-endorsed tests are available at specially negotiated low prices only to the public sector and import duties also add to the costs. In addition, financial incentives and laboratory margins further inflate the costs to make them virtually unaffordable to the average private sector patient.

This has now changed, thanks to a new initiative launched in March 2013, to improve the affordability of WHO-endorsed TB tests. Initiative for Promoting Affordable, Quality TB tests (IPAQT; www.ipaqt.org) is a coalition of private labs in India, supported by industry and nonprofit groups (e.g., Clinton Health Access Initiative), 
that has made WHO-endorsed tests available at affordable prices to patients in the private sector. ${ }^{[15]}$

IPAQT aims to facilitate the delivery of WHO-endorsed tests to the TB patient at affordable prices and promote the use of WHO-endorsed TB tests by building awareness about these new, validated/endorsed tests among health providers, laboratories, and patients. Several private laboratories in India have agreed that in exchange for not exceeding negotiated, ceiling prices to patients, notifying the government of the cases diagnosed, promoting the use of these tests, and participating in external quality assurance they would get reagents at significantly reduced prices. In exchange for offering lower prices, the manufacturers and distributors would receive greater and more predictable volumes from the previously untapped private market.

The business model of IPAQT is based on a comparison of high margin low volume (premium) versus lower margin high volume (mass-market) pricing models. Thanks to IPAQT, the cost of Xpert MTB/RIF is now reduced to Rs. 1700 (maximum price labs can charge patients). The line probe assay (Hain Genotype MTBDRplus Version 2) is now available at Rs. 1600 . These prices are approximately
$50 \%$ less than the private market prices before IPAQT was launched, and the prices are comparable to the banned TB ELISA test for three antibodies. Thus, for the money patients were paying for inaccurate tests, they can now get WHO-endorsed, high-quality tests.

Laboratories in IPAQT will soon offer other WHO-endorsed tests (e.g., liquid cultures) at transparently advertised, negotiated, lower prices. TB cases diagnosed will be notified to the RNTCP for linkages to free TB drugs, where necessary. Any Indian laboratory can join IPAQT, provided they are accredited by a recognized agency (e.g., National Accreditation Board for Testing and Calibration Laboratories) and agree to abide by the guiding principles of IPAQT. Laboratories that join IPAQT must agree to stop doing TB serology and avoid promoting tests (e.g., IGRAs) that are discouraged by the RNTCP.

Since its launch in March of 2013, the IPAQT initiative has already achieved a pan-India presence-with 42 labs which encompasses over 3000 franchisee labs and greater than 10,000 collection centers committed to providing these tests at affordable prices [Figure 2]. The number of labs is expected to increase significantly in the

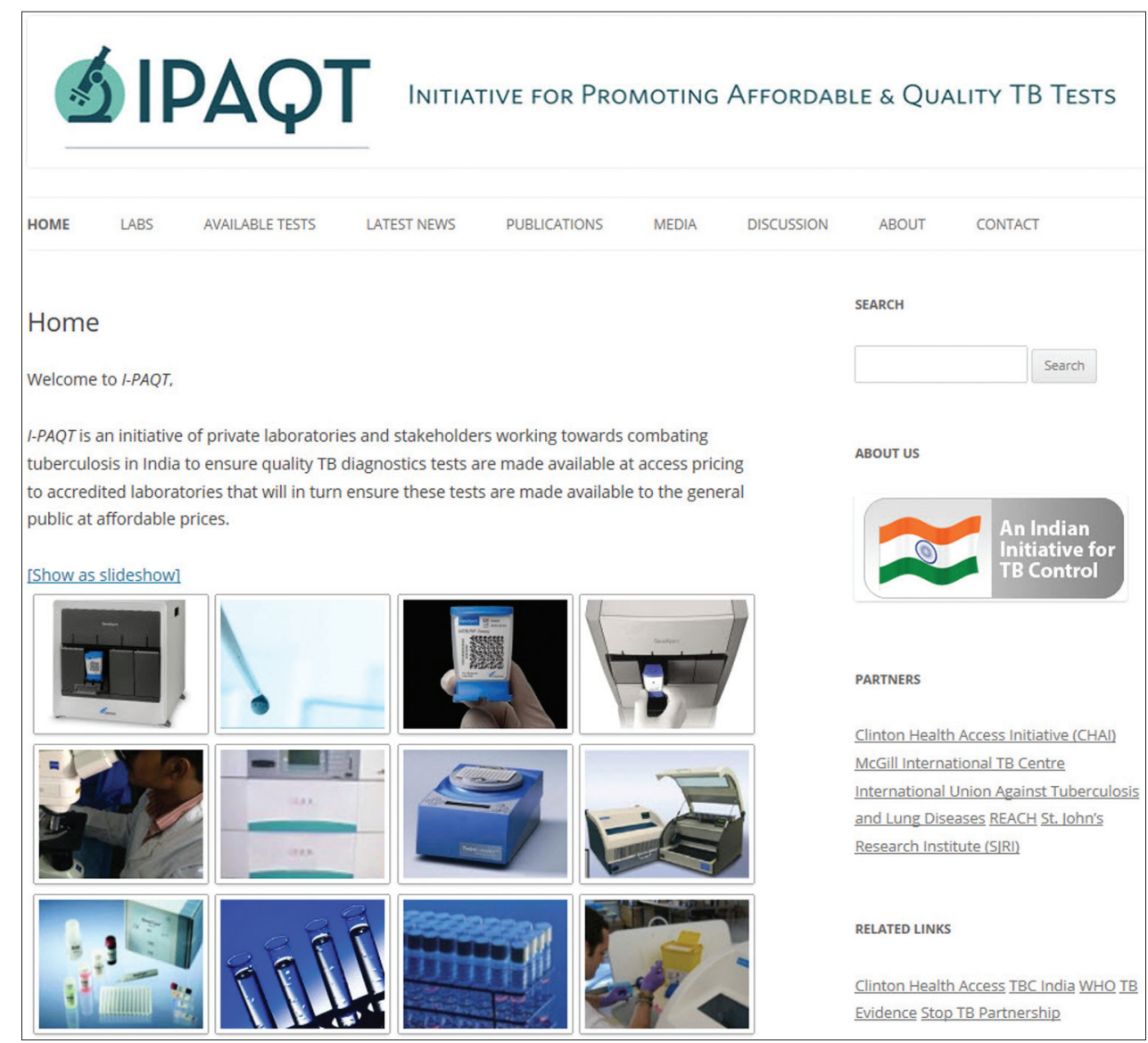

Figure 2: Web site of the initiative for promoting affordable, quality TB tests initiative (www.ipaqt.org) 
months ahead. Thus, this initiative is expected to greatly increase affordability for private sector patients and improve the quality of TB care in the country. In the long run, removal of import duties for all WHO-endorsed TB tests (under lifesaving drugs exemption) along with encouraging domestic development of high-quality TB tests will be critical to achieving the RNTCP goal of universal access.

\section{Madhukar Pai}

McGill International TB Centre and Department of Epidemiology and Biostatistics, McGill University, Montreal, Canada

\section{Address for correspondence: Dr. Madhukar Pai, E-mail: madhukar.pai@mcgill.ca}

\section{REFERENCES}

1. Udwadia ZF. MDR, XDR, TDR tuberculosis: Ominous progression. Thorax 2012;67:286-8.

2. Sachdeva KS, Kumar A, Dewan P, Satyanarayana S. New Vision for Revised National Tuberculosis Control Programme (RNTCP): Universal access-"Reaching the un-reached". Indian J Med Res 2012;135:690-4.

3. Kapoor SK, Raman AV, Sachdeva KS, Satyanarayana S. How Did the TB patients reach DOTS services in Delhi? A study of patient treatment seeking behavior. PLoS One 2012;7:e42458.

4. Satyanarayana S, Nair SA, Chadha SS, Shivashankar R, Sharma G, Yadav S, et al. From where are tuberculosis patients accessing treatment in India? Results from a cross-sectional community based survey of 30 districts. PLoS One 2011;6:e24160.

5. Jarosawlski S, Pai M. Why are inaccurate tuberculosis serological tests widely used in the Indian private healthcare sector? A root-cause analysis.
J Epidemiol Glob Health 2012;2:39-50.

6. Udwadia ZF, Pinto LM, Uplekar MW. Tuberculosis management by private practitioners in Mumbai, India: Has anything changed in two decades? PLoS One 2010;5:e12023.

7. Singh S, Katoch VM. Commercial serological tests for the diagnosis of active tuberculosis in India: Time for introspection. Indian J Med Res 2011;134:583-7.

8. Steingart KR, Flores LL, Dendukuri N, Schiller I, Laal S, Ramsay A, et al. Commercial serological tests for the diagnosis of active pulmonary and extrapulmonary tuberculosis: An updated systematic review and meta-analysis. PLoS Med 2011;8:e1001062.

9. World Health Organization. Policy statement: Commercial serodiagnostic tests for diagnosis of tuberculosis. In Geneva: World Health Organization; 2011.

10. World Health Organization. Policy statement: Use of tuberculosis interferon-gamma release assays (IGRAs) in low- and middle-income countries. In Geneva: World Health Organization; 2011.

11. Metcalfe JZ, Everett CK, Steingart KR, Cattamanchi A, Huang L, Hopewell PC, et al. Interferon-gamma release assays for active pulmonary tuberculosis diagnosis in adults in low- and middle-income countries: Systematic review and meta-analysis. J Infect Dis 2011;204(Suppl 4):S1120-9.

12. World Health Organization. Policy statement: Automated real-time nucleic acid amplification technology for rapid and simultaneous detection of tuberculosis and rifampicin resistance: Xpert MTB/RIF system. In Geneva: World Health Organization; 2011.

13. Steingart KR, Sohn H, Schiller I, Kloda LA, Boehme CC, Pai M, et al. Xpert ${ }^{\circledR}$ $\mathrm{MTB} / \mathrm{RIF}$ assay for pulmonary tuberculosis and rifampicin resistance in adults. Cochrane Database Syst Rev 2013;1:CD009593.

14. Policy statement. Molecular line probe assays for rapid screening of patients at risk of multidrug-resistant tuberculosis (MDR-TB). World Health Organization, 2008. Available from: http://www.who.int/tb/laboratory/ policy_statements/en/index.html [Last accessed on 2013 Jun 17].

15. Kay M. Private firms form initiative to offer accurate and affordable TB tests. BMJ 2013;346:f2161.

How to cite this article: Pai M. Promoting affordable and quality tuberculosis testing in India. J Lab Physicians 2013;5:1-4.

Announcement

\section{“QUICK RESPONSE CODE” LINK FOR FULL TEXT ARTICLES}

The journal issue has a unique new feature for reaching to the journal's website without typing a single letter. Each article on its first page has a "Quick Response Code". Using any mobile or other hand-held device with camera and GPRS/other internet source, one can reach to the full text of that particular article on the journal's website. Start a QR-code reading software (see list of free applications from http://tinyurl.com/yzlh2tc) and point the camera to the QR-code printed in the journal. It will automatically take you to the HTML full text of that article. One can also use a desktop or laptop with web camera for similar functionality. See http://tinyurl.com/2bw7fn3 or http://tinyurl.com/3ysr3me for the free applications. 